\title{
Les besoins des personnes âgées : une construction sociohistorique mouvante
}

Évolution des discours et pratiques liés à la prise en charge de la vieillesse (canton de Vaud, $\mathrm{XIX}^{\mathrm{e}}-\mathrm{XXI}^{\mathrm{e}}$ siècles)

\section{Séverine Pilloud et Cécilia Bovet}

\section{(2) OpenEdition}

Édition électronique

URL : http://journals.openedition.org/hms/1200

DOI : $10.4000 / \mathrm{hms} .1200$

ISSN : 2557-2113

\section{Éditeur}

Presses universitaires du Midi

\section{Édition imprimée}

Date de publication : 17 mai 2018

Pagination : 151-170

ISBN : 978-2-8107-0542-9

ISSN : 2263-8911

Ce document vous est offert par Centre national de la recherche scientifique (CNRS)

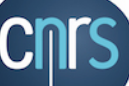

Référence électronique

Séverine Pilloud et Cécilia Bovet, «Les besoins des personnes âgées : une construction

sociohistorique mouvante », Histoire, médecine et santé [En ligne], 12 I hiver 2017, mis en ligne le 28

mai 2018, consulté le 04 juillet 2018. URL : http://journals.openedition.org/hms/1200 ; DOI : 10.4000/ hms. 1200 


\title{
Les besoins des personnes âgées : une construction sociohistorique mouvante Évolution des discours et pratiques liés à la prise en charge de la vieillesse (canton de Vaud, XIXe-XXI siècles)
}

\author{
Séverine Pilloud
}

Professeure ordinaire à la Haute École de la Santé La Source (Lausanne)

\section{Cécilia Bovet}

Adjointe scientifique à la Haute École de la Santé La Source (Lausanne)

Les modes de prise en charge de la vieillesse ont connu de considérables changements au cours du temps. Les mesures sociales et sanitaires mises en place pour répondre aux problématiques soulevées par le sort des personnes âgées sont principalement déterminées par les images sociales prégnantes de cette catégorie de la population. Il s'agit d'examiner la manière dont ses besoins spécifiques sont appréhendés, en fonction des caractéristiques socioéconomiques des individus et de leur état de santé. Par l'analyse de l'histoire des représentations sur le grand âge dans le canton de Vaud (Suisse romande) entre la fin du XIX ${ }^{e}$ siècle et le début du Xxi ${ }^{\text {e }}$ siècle, cet article vise à étudier les transformations des rapports entre la société et ses aînés. Cette évolution renvoie à des modifications sociales, culturelles, économiques et politiques qui permettent de réfléchir aux problématiques actuelles relatives au vieillissement démographique et aux enjeux concernant le devenir des systèmes sanitaires.

Mots-clés : vieillesse, représentations, besoins, institutions pour personnes âgées

Welfare and healthcare for old people went through important changes during history. Social practices designed to meet the issues raised by old age are mainly determined by the prevalent images of the elderly. It is of most interest to look into the way their specific social and caring needs are envisioned, taking notably into account their socioeconomic background and their health. Analyzing the evolution of dominant representations of old age in the canton of Vaud (French-speaking Switzerland) between the end of the XIXth century and the beginning of the XXIth century, this article aims at studying the transformations of relationships between society and old people. This historical process sheds light on the influences played by major modifications at the sociocultural, economic and political levels. Such results allow to think about the current problems relative to the demographic ageing and to the contemporary stakes concerning the becoming of sanitary institutional systems.

Keywords: old age, representations, needs, nursing homes

Séverine Pilloud et Cécilia Bovet, « Les besoins des personnes âgées : une construction sociohistorique mouvante », Histoire, médecine et santé, I2, hiver 20I7, p. I5I-I70. 


\section{Introduction}

Le vieillissement de la population, très marqué depuis le début du $\mathrm{xx}^{\mathrm{e}}$ siècle en Occident et dans une majorité de pays développés, a donné lieu à de nombreux travaux de recherche en sciences humaines et sociales dans lesquels on s'attache notamment à anticiper les structures institutionnelles à mettre en place pour répondre à cette nouvelle donnée démographique ${ }^{\mathrm{I}}$. Or, la question des besoins spécifiques des personnes âgées est fréquemment utilisée comme si ces dernières représentaient une catégorie n'ayant guère besoin d'être définie, leurs besoins étant considérés dans une perspective pratiquement essentialiste et transhistorique. Cependant, l'étude que nous avons réalisée sur l'histoire de la prise en charge sociosanitaire des personnes âgées dans le canton de Vaud, depuis la reconnaissance sociopolitique du problème du vieillissement à partir de seconde moitié du XIX $x^{\mathrm{e}}$ siècle, démontre à quel point les besoins des aînés ${ }^{2}$ sont loin de constituer une réalité figée'. Au contraire, il s'agit bien de perceptions fortement inscrites dans un contexte socio-économique, culturel et historique. Ces visions sont en outre susceptibles de varier en fonction des caractéristiques sociodémographiques des divers acteurs et actrices qui les formulent, notamment en fonction de la position de ceux-ci dans le système de santé en charge des populations vieillissantes ${ }^{4}$. Les modes d'appréhension des besoins des personnes âgées sont donc sujets à de fortes évolutions. De fait, les institutions et les mesures qui se sont mises en place au fil du temps dans le domaine de l'accompagnement de la population vieillissante sont elles-mêmes gouvernées par des logiques propres. Elles suivent en cela aussi leurs besoins respectifs, lesquels n'échappent pas aux variations sociohistoriques et culturelles5. Par l'analyse de

\footnotetext{
I | François Höpflinger et Valérie Hugentobler, Les besoins en soins des personnes âgées en Suisse : prévisions et scénarios pour le XXI siècle, Berne, H. Huber/Observatoire suisse de la santé, 2003.

2 | Le masculin est utilisé ici par commodité et comprend également le féminin.

$3 \mid$ « Histoire de la prise en charge des personnes âgées dans le canton de Vaud entre la fin du $\mathrm{XIX}^{\mathrm{e}}$ siècle et la seconde moitié du XXe siècle : évolution de la perception des besoins socio-sanitaires et des structures institutionnelles ", recherche financée par le Fonds national suisse (FNS) et la Fondation Leenaards (20II-20I3). Requérante principale : Dr. Séverine Pilloud ; co-requérant : Dr. Vincent Barras ; adjointe scientifique : Cécilia Bovet.

4 | Elise Feller, Histoire de la vieillesse en France: 1900-1960, du vieillard au retraité. Paris, Arslan, 2005; Alain Pilon, « La vieillesse : reflet d'une construction sociale du monde », Nouvelles pratiques sociales, 3/2, 1990, p. I4I-156 ; Stefano Cavalli, Établissements pour personnes âgées et construction sociale de la vieillesse, mémoire de licence en sociologie, université de Genève, 1997 ; id., Vieillards à domicile, vieillards en pension : une comparaison, Lausanne, Réalités sociales, 2002 ; Bernard Arcand, « La construction culturelle de la vieillesse », Anthropologie et Sociétés, 6, 1982, p.7-23.

5 | Patrice Bourdelais, "L'âge de la vieillesse, l'histoire d'un progrès ", dans Anne Nardin (éd.), Voyages au pays de Gérousie. Le grand âge en institution, Paris, Musée de l'assistance publique et Hôpitaux de Paris, p. 20-24; id. et Olivier Faure, Les nouvelles pratiques de santé : objets, acteurs, pratiques sociales, $X V I I I^{e}-\mathrm{XX}{ }^{e}$ siècles, Paris, Belin (Histoire et société), 2005.
} 
l'histoire des discours sur le grand âge entre la fin du XIx $x^{\mathrm{e}}$ siècle et le début du $\mathrm{XXI}^{\mathrm{e}}$ siècle, cet article vise à examiner de manière critique les transformations qui se sont produites dans la manière d'appréhender les personnes âgées et leurs nécessités spécifiques sur les plans social et sanitaire.

\section{Approche et méthodologie}

Notre questionnement principal visait à mettre en lumière les changements majeurs intervenus depuis la seconde moitié du XIX siècle en ce qui concerne la définition des besoins des personnes âgées. À quels moments les discours se transforment-ils ? Comment ces modifications sont-elles perceptibles dans les sources analysées ? Qui sont les principaux porteurs de nouvelles visions du vieillissement? Dans quel contexte sociohistorique et culturel ces modifications des représentations s'inscrivent-elles ? En quoi reflètent-elles des mutations macrosociales plus larges?

Notre recherche a d'abord consisté à dépouiller un corpus principal d'archives : le fonds privé de La Rozavère ${ }^{6}$, premier établissement médico-social (EMS) du canton de Vaud créé en I887. Les archives de cette institution sont composées de procès-verbaux, rapports annuels, correspondance, registres, règlements, publications jubilaires, plans d'architecture et photographies. Quand le premier établissement ouvre, une vingtaine de vieillards (un peu plus d'hommes que de femmes) sont accueillis. Jusqu'au milieu du $x^{\mathrm{e}}$ siècle, les médecins, qui viennent en moyenne un jour par semaine, offrent leurs visites et traitements. La directrice et ses aides (six au début) assurent le reste des soins. Un comité, composé de douze membres (médecins, juges et professeurs, banquiers, représentants religieux) assure la gestion. Ce sont eux qui signent la grande majorité des documents consultés. Ce corpus a été mis en perspective avec d'autres archives institutionnelles (EMS, hospices cantonaux, etc.) exploités à titre de comparaison (régions urbaines/régions rurales ; territoires protestants/territoires catholiques).

À l'aide d'une base de données créée dans l'objectif de recueillir tous les éléments de discours ainsi que les diverses étapes de mutation institutionnelle traversées par La Rozavère, une méthodologie inspirée de la Grounded Theory et de l'anthropologie historique a été utilisée 7 . Il s'est agi de restituer, de manière littérale et sans produire d'anachronismes, les représentations et les pratiques à

6 | Établissement de 128 lits, situé au Chemin de Rovéréaz 23, dans le quartier de Chailly à Lausanne, fondation de droit privé. Nous remercions chaleureusement la direction de La Rozavère qui s'est montrée ouverte et enthousiaste et nous a permis de passer le temps nécessaire à cette recherche. 7 | Clifford Geertz, "La description dense. Vers une théorie interprétative de la culture », Enquête. Anthropologie, histoire, sociologie, 6, 1998, p. 73-I05 ; Anselm Strauss et Juliet Corbin, Les fondements 
l'égard des personnes âgées en restant au plus près du point de vue des différents acteurs sociaux impliqués dans cette histoire institutionnelle de plus de 125 ans. Les données ont été traitées de manière diachronique et analysées de façon thématique, afin de faire émerger les changements de perception. Parallèlement, dans une perspective d'histoire sociale, les transformations discursives ont été contextualisées par rapport aux principaux déterminants sociohistoriques et culturels dans lesquelles elles s'inscrivent et qui impactent nécessairement tant sur la construction des besoins des personnes âgées que sur les mesures institutionnelles qui ont été proposées pour s'efforcer d'y répondre. La méthode de l'histoire nécessite une élucidation du genre de source à analyser et de l'audience qu'elle concerne, ainsi que des intérêts et enjeux propres aux acteurs et actrices qui s'y prononcent. Pour cette raison, les archives publiques ont été largement consultées ${ }^{8}$, afin d'avoir accès au discours des politiques et des autorités sanitaires, tout en permettant de documenter la situation qui prévalait avant la construction des premiers asiles pour vieillards dans le canton de Vaud.

\section{Situation des «vieillards" au XIX ${ }^{\mathrm{e}}$ siècle avant la création des premières institutions}

Avant la naissance des premiers asiles pour la vieillesse sur le continent européen dans les dernières décennies du XIX siècle, la plupart des « vieillards » font partie de la catégorie des "indigents »9 . En l'absence d'un système de sécurité sociale étendu à tous, les individus que le grand âge empêche de travailler se retrouvent démunis ou dépendants de leur cercle familial pour survivre, excepté la minorité d'entre eux qui sont suffisamment fortunés pour vivre grâce à une rente $^{\mathrm{io}}$. Ce sont avant tout leurs besoins en termes de revenus économiques qui sont soulignés dans les archives ${ }^{\mathrm{II}}$. À une époque où le travail productif est fortement valorisé, la personne âgée peut également être victime d'exclusion sociale,

de la recherche qualitative. Techniques et procédures de développement de la théorie enracinée, Fribourg, Academic Press Fribourg/Éditions Saint-Paul, 2004.

$8 \mid$ Archives cantonales vaudoises (ACV), Fonds Secours publics, KVIII a, b et d.

9 | Jean-Pierre Bois, «La restriction des libertés chez les personnes vulnérables. Le point de vue de l'histoire » Gérontologie, II4, 2000, p. 20-25.

Io | Christian Lalive d'Épinay, «La construction des parcours de vie et de la vieillesse en Suisse au cours du Xx ${ }^{e}$ siècle ", dans Geneviève Heller (éd.), Le poids des ans. Une histoire de la vieillesse en Suisse romande, Lausanne, Éditions d'en bas, 1994, p. 127-150 ; Feller, Histoire de la vieillesse en France..., op. cit. ; Geneviève Heller, « De l'asile à l'établissement médico-social : le Canton de Vaud, fin XIxXx ${ }^{e}$ siècle ", dans ead. (éd.), Le poids des ans, op. cit., p. II3-I26 ; David Thomson, « The welfare of the elderly in the past: A family or community responsibility ", dans Margaret Pelling et Richard M. Smith (éd.), Life, Death, and the Elderly: Historical Perspectives, Londres, Routledge, I991, p. 194-22I. II | Jean-Pierre Bois, «Âge, pauvreté ou richesse. Observation historique sur la question des vieux et de l'argent », Gérontologie et société, II7/2, 2006, p. 15-30. 
dans la mesure où elle ne participe plus à la croissance ${ }^{\mathrm{I} 2}$. Les besoins sur le plan de l'intégration sociale ne sont pas décrits en ces termes, bien que nombre d'archives de l'époque mentionnent l'isolement dans lequel se retrouvent des sujets sans famille ou éloignés de celle-ci ${ }^{13}$.

Dans un tel contexte, l'aide est destinée à des vieillards en situation de grande précarité financière et privés d'un cercle familial pouvant faire office de soutien. Les éventuels besoins en matière de santé demeurent subsidiaires, même si les discours commencent à différencier les vieillards infirmes de ceux qui sont suffisamment valides pour accomplir certaines tâches en échange des prestations qu'ils reçoivent ${ }^{14}$. Ces modalités d'aide recouvrent des modes de prises en charge qui tendent à se superposer : les hospices ou hôpitaux généraux, qui constituent des lieux d'internement pour toutes formes de détresse sociale ${ }^{\mathrm{i} 5}$, et les dépôts de charité ou des ateliers de travail en milieu communautaire, qui accueillent en majorité une population âgée ${ }^{16}$. Avec la médicalisation progressive des hôpitaux publics, les services hospitaliers commencent à axer prioritairement leur mission sur le traitement de maladies aiguës ${ }^{17}$. Les directions hospitalières se trouvent dans l'obligation de se décharger des « incurables », une catégorie dans laquelle on inclut la vieillesse fragilisé $\mathrm{e}^{\mathrm{I}}$. Ces cas incurables ou chroniques sont progressivement vus comme des patient.e.s qui encombrent les lits hospitaliers et pour lesquels d'autres formes d'aide doivent être imaginées.

Dans le canton de Vaud par exemple, l'hospice cantonal créé en I806 regroupe une population très hétérogène, qui comprend quelques malades, mais aussi des aliéné·e·s, des détenu·e·s, des infirmes, des vagabond·e·s ainsi que des vieillards ${ }^{19}$. Une population métissée que la collectivité a besoin de mettre

I2 | Jean-Pierre Bois, «Exclusion et vieillesse. Introduction historique », Gérontologie et société, I02/3, 2002, p. 13-24.

I3 | Yannick Marec et Daniel Réguer, De l'hospice au domicile collectif: la vieillesse et ses prises en charge de la fin du xVIII e siècle à nos jours, Mont-Saint-Aignan, Presses universitaires de Rouen et du Havre, 2013.

I4 | Séverine Pilloud, « La thérapeutique par le travail dans le traitement des maladies mentales : l'asile cantonal de Cery (Prilly-Lausanne) I873-I899 ", Revue médicale de la Suisse romande, II6, I996, p. 877-886.

I5 | Olivier Faure, Genèse de l'hôpital moderne. Les hospices civils de Lyon de I802 à I845, Lyon, Presses universitaires de Lyon, 1982 ; Francis Stéfanini, Un demi-siècle de vie hospitalière en Savoie I860-I9I4: charnière entre tradition et modernité, thèse d'histoire sous la direction d'Olivier Faure, Université Jean-Moulin, Lyon, 1998.

I6 | Feller, Histoire de la vieillesse en France..., op. cit.

I7 | Pierre-Yves Donzé, Bâtir, gérer, soigner. Histoire des établissements hospitaliers de Suisse romande, Chêne-Bourg/Genève, Georg éditeur, 2003.

I8 | Séverine Pilloud, Julie Hofmann. Une vie de combat auprès des exclus, Lausanne, Fondation Eben Hézer, 1998.

I9 | Séverine Pilloud, «La thérapeutique par le travail... », art. cité; Heller, «De l'asile à l'établissement médico-social... ", art. cité. 
à l'écart et de surveiller dans une perspective de contrôle social ${ }^{20}$. Les exigences de la société tout autant que celles des institutions hospitalières priment sur la nécessité d'élaborer une prise en charge adaptée pour ces différents groupes sociaux. Ainsi, en I8IO, un projet de décret vaudois vise à créer un établissement de trente lits pour incurables et vieillards infirmes, mais il s'agit alors moins de résoudre les besoins propres à cette catégorie de la population que de décharger l'hospice des pensionnaires âgés ou invalides, afin de répondre aux besoins thérapeutiques des malades curables ${ }^{21}$. Cette motion sera toutefois abandonnée au profit d'autres priorités mises en avant par les politiques : un asile pour aliéné·e·s, ouvert en I8II, et, quinze ans plus tard, un pénitencier pour les détenus.

C'est finalement une initiative privée qui palliera, dès 1827 , l'absence de décisions publiques en faveur de la population vieillissante : une "Société en faveur des incurables » se fonde à Lausanne sous la houlette d'une association de bienfaisance présidée par des pasteurs et l'élite sociale ${ }^{22}$. Le canton de Vaud est alors dirigé par les ténors du parti libéral, qui préfèrent laisser les œuvres de charité aux particuliers ou à l'Église plutôt que de mettre en place une assistance légale étatique ${ }^{23}$. Cette entreprise en faveur des « incurables » cherche à répondre à des besoins que le terme même d'incurable reflète bien : étant donné que les besoins médicaux sont perçus comme inutiles dans la mesure où on ne pourrait pas guérir du vieillissement ou des pathologies qui y sont liées - ellesmêmes d'ailleurs encore peu connues puisque la gériatrie en est encore à ses balbutiements ${ }^{24}-$, on se contente de prévoir un système de placement dans des familles d'accueil pour les vieillards privés de secours financiers ou familiaux. Cet organisme fonctionnera pendant plusieurs années comme l'unique mode de soutien aux vieillards du canton. Les besoins de ceux-ci sont décrits en termes succincts : il leur faut un habitat au sein d'un cercle familial capable de répondre aux impératifs vitaux, à savoir manger et dormir dans un environnement sain ${ }^{25}$.

Plusieurs événements contribueront à modifier la donne dès le milieu du XIX ${ }^{\mathrm{e}}$ siècle. La publication, en I84I, d'une enquête sur le paupérisme, demandée par le Grand Conseil vaudois, met en évidence la vieillesse comme facteur

20 | Andrée Gautier, «L'asile départemental du Perron dans l'Isère (I892-I960) », Gérontologie, II5, 2000, p. 44-5I.

2I | ACV, Fonds Secours publics, KVIII aI.

$22 \mid$ Ibid.

23 | Bruno Dumons, «Vieillesse et "État providence" en Suisse romande dans la première moitié du xx $x^{e}$ siècle ", Le mouvement social, 190, 2000, p. 9-3I ; Jean-Pierre Tabin, Arnaud Frauenfelder, Carola Togni et Véréna Keller, Temps d'assistance. Le gouvernement des pauvres en Suisse romande depuis la fin $d u$ XIX $X^{e}$ siècle, Lausanne, Antipodes, 2008.

24 | Vincent Barras, «Histoire du médecin de I880 à la fin du Xxe siècle ", dans Louis Callebat (dir.), Histoire du médecin, Paris, Flammarion, 1999, p. 269-307.

25 | ACV, Fonds Secours publics, KVIII aI. 
premier de pauvreté ${ }^{26}$. Si io \% de la population vaudoise vit dans des conditions proches de la misère, dans une économie essentiellement agricole et rurale, le sort des vieillards s'avère particulièrement inquiétant. Nombre d'entre eux sont réduits à la mendicité et au vagabondage, des comportements qui troublent par ailleurs l'ordre social ${ }^{27}$. La précarité régnante favorisera la prise de pouvoir progressive des radicaux sur les libéraux entre $\mathrm{I} 845$ et I848, ce qui conduira à une participation accrue de l'État de Vaud dans les structures d'assistance ${ }^{28}$. Le parti radical prône en effet des réglementations étatiques concernant les populations fragilisées, préludant à l'instauration d'un état plus social et attentif au sort des vieillards dans la société.

En I850, les autorités vaudoises créent un organe "destiné au soulagement des malades déclarés incurables et des vieillards infirmes ${ }^{29}$ ». Il s'agit encore d'un système de placement dans des familles mais destiné à un nombre plus grand de candidats et prévoyant des aides sous la forme de pensions mensuelles pour les personnes âgées ou de biens en nature : vêtements, chaussures, couverture, etc. Cette institution de « charité publique » ambitionne de " pourvoir aux besoins des personnes appartenant à cette classe malheureuse de la société, qui, privées de toute ressource, ne peuvent, à raison de leur âge avancé ou de leurs infirmités, subvenir à leur existence $»^{30}$. Il est avant tout fait référence à des «secours sociaux » pour ceux qui auraient perdu leurs moyens de subsistance. Très rares sont les mentions de soins ou de traitements, même si l'on évoque de temps à autre quelques remèdes de base. De manière générale, il s'agit toujours d'une prise en charge visant l'entretien de la vie : nourrir, loger et vêtir. Au milieu du XIX ${ }^{\mathrm{e}}$ siècle, les autorités vaudoises estiment que ce système de pensions financières et de placement familial est suffisant ; du moins correspond-il aux priorités politiques et économiques, ainsi qu'aux valeurs socioculturelles : les représentants politiques insistent sur le devoir d'assistance ${ }^{3 \mathrm{I}}$ (aussi bien moral que contenu dans le Code civil) qui incombe aux membres de la famille ${ }^{32}$.

L'objectif principal des placements familiaux est d'offrir un cadre sécurisé " qui permet [aux vieillards] d'achever en paix leur passage dans ce monde ${ }^{33}$ ». La vieillesse est envisagée comme une étape de vie dont l'horizon indépassable est une mort prochaine, à laquelle les vieillards ne peuvent se préparer que par

26 | ACV, Bulletin du Grand Conseil, vol. 4I, I850.

27 | Heller, « De l'asile à l'établissement médico-social... », art. cité.

28 ACV, Bulletin du Grand Conseil, vol. 4I, I850.

$29 \mid$ Ibid.

30 | ACV, Fonds Secours publics, KVIII aI.

3I $\mid$ Ibid.

32 Heller, Le poids des ans, op. cit.

33 ACV, Fonds Secours publics, KVIII aI. 
le soutien religieux des pasteurs. Les besoins des personnes âgées en termes de qualité de vie ne sont pas davantage explicités, encore moins leurs droits, à une époque où la société est encore relativement contraignante pour le commun des mortels. Par contre, les devoirs qui leur incombent sont régulièrement spécifiés. L'aide se trouve en effet conditionnée à une obligation de «bonne conduite ${ }^{34}$ »; les archives des secours publics attestent de plusieurs cas de suspension des pensions financières « pour cause d'ivrognerie, d'insubordination, de vagabondage, d'insultes ou de mendicité ${ }^{35}$ ». Autant de termes qui attestent de la vision paternaliste et relativement autoritaire qui domine dans l'attitude des élites par rapport à la population assistée et aux vieillards en particulier. Les mesures de soutien social sont accompagnées de formes de surveillance et de normalisation. On craint en particulier les éventuels débordements des hommes seuls, issus de couches sociales défavorisées, et dont les excès d'alcool ou l'oisiveté forcée peuvent amener à créer du désordre dans la collectivité ${ }^{36}$.

Ce souci de l'ordre public a sous-tendu les débats relatifs à la création des premiers asiles, renvoyant à la dimension ségrégative de ces établissements ${ }^{37}$. Dès I852, les édiles cantonaux évoquent, dans un document interne de la commission des secours publics du canton de Vaud qui ne circulera pas en dehors du cercle des parlementaires, la possibilité de subventionner un hospice fermé qui servirait avant tout à retrancher de la vie sociale des individus dont l'état de santé devient trop problématique pour les familles qui doivent s'en charger :

\footnotetext{
[À propos du projet de] créer des établissements spéciaux destinés aux maladies dégoûtantes [...] il y a déjà quelques malades qui n'ont été placés chez des particuliers qu'avec beaucoup de peine et pour lesquels un établissement de ce genre serait à désirer. Quoique le moment de s'occuper de la création de ces établissements ne soit pas encore venu, [...] on peut invoquer son utilité [...] pour les malades dont la présence dans leur famille ou dans la société est un objet de dégoût et d'effroi ${ }^{8}$.
}

Cet aspect de mise à l'écart ne saurait toutefois faire oublier une autre fonction potentielle et cruciale de l'asile, celle de protection. Une dizaine d'années plus tard, en I863, un médecin formule un discours qui met en avant la situation des vieillards et présente l'hospice de vieillards non pas comme un lieu d'exclusion mais en tant que refuge. Dans son Mémoire sur l'utilité d'un asile en faveur de la vieillesse indigente du canton de Vaud, le médecin Vullyamoz-Blanc dénonce les limites de l'aide apportée jusqu'ici et propose une hiérarchisation des secours nécessaires aux personnes âgées :

34 | ACV, Fonds Secours publics, KVIII a67/I3.

35 | ACV, Fonds Secours publics, KVIII a67/II.

36 | ACV, Fonds Secours publics, KVIII aI.

$37 \mid$ Ibid.

38 | Ibid. 
L'expérience m'a montré que les vieillards sont souvent abandonnés [...]. Il faut au vieillard de la compagnie, des secours religieux, des soins chirurgicaux, qui ne sont pas toujours à sa portée lorsqu'il est isolé dans nos campagnes [...] Mis en pension, le vieillard est arraché à ses habitudes, en proie aux regrets que lui cause l'éloignement de sa famille; la tristesse et l'ennui l'accablent, s'ajoutent aux chagrins et aux maux de son âge avancé. Dans cette position, le vieillard plus que tout autre a besoin d'un ami du même âge, soumis aux mêmes épreuves, seul capable de comprendre ses plaintes et de ressentir une vraie sympathie $[\ldots]^{39}$.

Les apports de l'asile sur le plan de la sociabilité ainsi que les soins médicaux sont évoqués dans ces propos, avec une attention nouvelle sur les difficultés psychologiques que peuvent induire le placement dans une famille d'accueil et la perte de son ancien mode de vie. Toutefois, lorsqu'il liste les nécessités des vieillards, le Dr Vullyamoz-Blanc place en premier lieu les « besoins religieux », à une époque où les valeurs chrétiennes imprègnent nombre d'œuvres caritatives, dans un canton où l'élite sociale est majoritairement représentée par de fervents protestants ${ }^{40}$. En seconde position viennent les « soins hygiéniques ", qui ne détaillent aucune prestation sanitaire mais plutôt une amélioration des conditions d'existence au niveau de la propreté, de l'aération et de l'hygiène de l'habitat. Précisons à ce titre qu'un grand nombre de cas de négligence - sans que le terme de maltraitance ne soit explicitement articulé - au détriment des vieillards ont en effet été relevés dans les milieux ruraux, comme en témoignent les archives des secours publics ${ }^{41}$. Pour plusieurs familles désargentées, l'hébergement de vieillards est envisagé comme une source de gain fort utile, et les hôtes les moins scrupuleux n'hésitent pas à exploiter cette main-d'œuvre fragile tout en lui réservant un abri déplorable. Une veuve est par exemple dénoncée à la suite de la visite d'un pasteur en 1870 ; elle logeait quatre pensionnaires dans une petite chambre sans chauffage en échange de multiples tâches à son service ${ }^{42}$. Un paysan a quant à lui fait dormir un pensionnaire dans une écurie ${ }^{43}$. Comme en atteste un bulletin du Grand Conseil Vaudois, il existe même une pratique de "Mise publique des vieillards et des enfants » dans certaines régions agraires, une forme de marchandage au détriment des groupes sociaux vulnérables que dénonce vigoureusement un député vaudois en I874:

Je proposerai d'inviter le Conseil d'État à présenter [...] un projet de dispositions législatives ayant pour but de protéger plus efficacement qu'ils ne le sont aujourd'hui les orphelins et les vieillards nécessiteux. [...] nous avons le regret de voir encore

39 | Vullyamoz-Blanc, cité dans Heller, Le poids des ans, op. cit., p. I22.

40 | Pilloud, Julie Hofmann, op. cit.

4I | ACV, Fonds Secours publics, KVIII aI.

42 ACV, Fonds Secours publics, KVIII a67/I6.

43 | Ibid. 
des communes annoncer dans les journaux et procéder publiquement à la mise des orphelins et des vieillards qui tombent à sa charge. C'est un abus fâcheux qu'il convient absolument de faire disparaître, car vous n'ignorez pas, Messieurs, que c'est ordinairement celui qui offre le moins de pain à ces malheureux qui obtient la préférence ${ }^{44}$.

De telles dénonciations conduiront à relancer les débats sur la création d'asiles pour vieillards à la fin du XIX ${ }^{\mathrm{e}}$ siècle. Cette période est en outre caractérisée par un vieillissement démographique marqué, grâce au recul progressif de la mortalité infectieuse et à la baisse de la natalité ${ }^{45}$. Progressivement, les vieillards devenus plus nombreux et plus âgés seront considérés non pas comme une sous-classe des indigents ou des incurables, mais comme une catégorie sociale en soi, ayant des besoins propres, notamment en fonction de leur degré de validité ${ }^{46}$. Les premiers asiles pour personnes âgées s'ouvrent dans les dernières décennies $\mathrm{du} \mathrm{XIX}^{\mathrm{e}}$ siècle ${ }^{47}$. Ils sont généralement destinés aux vieillards valides, privés de famille et modestes sur le niveau économique, les asiles ne souhaitant pas dispenser les descendants de leur devoir d'entretien à l'égard des aïeux ${ }^{48}$, ils se présentent donc comme une alternative en cas d'isolement et de pauvreté, sans pour autant promettre de soins médicalisés. L'hôpital fait office de recours temporaire ou définitif en cas d'altération de l'état de santé. Les soins au sein de l'asile sont donc limités à l'impératif de préservation de la vie. En I 887 , le règlement de La Rozavère, premier « asile pour vieillards pauvres et malheureux »(de son appellation initiale) ouvert sur le territoire vaudois, s'inscrit dans l'hygiénisme caractéristique de l'époque. Il affiche la volonté d'assurer un environnement sain, tant en ce qui concerne la salubrité des locaux que l'entretien des corps et la moralisation des esprits. La direction de l'institution entend fournir, dans l'ordre :

I. une bonne hygiène, basée sur une nourriture suffisante, saine et appropriée à des vieillards, sur la propreté personnelle, celle des chambres à coucher et des locaux communs ; sur des soins médicaux réguliers ; 2 la possibilité d'organiser, pour les vieillards valides, autant dans l'intérêt de leur santé que de leur moral, un travail proportionné à leurs forces et à leurs aptitudes ; 3. des distractions saines, qui pourraient

44 | ACV, Bulletin du Grand Conseil, séance du I8 mai, I874.

45 | Brigitte Santos-Eggimann, "Vieillissement et fragilité - comprendre pour prévenir ", dans Roxanne Currat et al., Au fil du temps. Le jeu de l'âge, Gollion, Infolio/Fondation Claude Verdan, 2008, p. 8I-85.

46 | Tabin, Frauenfelder, Togni et Keller, Temps d'assistance, op. cit.

47 | Heller, « De l'asile à l'établissement médico-social... », art. cité ; ead., Historique des maisons de retraite dans le canton de Vaud, rapport de recherche non publié, I994; ead., "Vieillir en institution depuis plus d'un siècle, l'exemple de l'asile de vieillards de Lausanne devenu La Rozavère ", Mémoire Vive, pages d'histoire lausannoise, 3, 1994, p. 9-17.

48 | Bois, « Âge, pauvreté ou richesse... », art. cité. 
avoir le plus heureux effet sur leur caractère et leurs sentiments ; 4 . une surveillance plus complète ; une influence morale plus directe et, par conséquent, plus effective ${ }^{49}$.

Le fait d'être occupé apparaît pour la première fois explicitement en tant que besoin. La réponse réside principalement dans le travail, lequel, avec sa dimension normalisatrice, correspond aux habitudes de l'époque, où les notions de temps libre et de loisirs ne sont pas entrées dans les mœurs, tant s'en faut ${ }^{50}$. Adopté dans nombre d'établissements réservés à des populations internées au XIX $x^{\mathrm{e}}$ siècle (aliénés, infirmes, orphelins), il représente à la fois un facteur d'ordre et d'économie, puisque les personnes internées collaborent aux tâches quotidiennes de l'institution ${ }^{51}$ : nettoyage, couture, préparation des repas ou réparations sommaires.

Le travail constitue également un dérivatif permettant de tromper l'ennui, comme dans beaucoup d'institutions sociomédicales qui se mettent en place à la même époque, notamment les asiles pour personnes présentant un handicap ${ }^{52}$. Si les distractions sont évoquées, elles se réduisent principalement à des cultes ou à des séances de lecture ; plus rarement les pensionnaires assistent aussi à des chantées offertes par des chœurs qui se rendent dans les murs de l'asile. Les individus accueillis dans l'établissement ont en effet rarement l'occasion d'en sortir, ou alors moyennant une permission spéciale, accordée au compte-gouttes par la direction. De fait, celle-ci admet que les vieillards ont besoin d'être surveillés, non pas en raison de pathologies

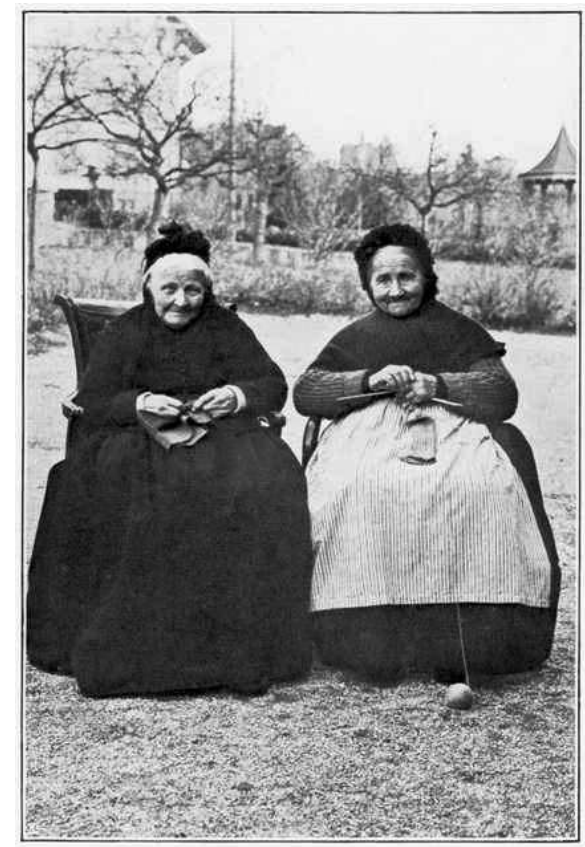

Femmes occupées à des travaux de couture Archives de La Rozavère (photo : de Jongh)

49 | Archives de La Rozavère, Règlement de l'Asile de vieillards pauvres et malheureux, I887.

50 | Pilloud, "La thérapeutique par le travail... », art. cité ; Sophie Richelle, « The Elderly Poor, or Poor Elderly. Old Age in Nursing Homes, Old Age on the Margins? », Journal of Belgian History, 46/I, 2016, p. 85-103; Mathilde Rossigneux-Meheaust, «L'invention d'un territoire de la vieillesse à Paris au XIX ${ }^{e}$ siècle. Construction et expérimentation d'une politique spatiale différenciée de l'assistance parisienne ", Histoire urbaine, 42/I, 2015, p. I43-I6I ; Sophie, Richelle et Iris Loffeier, « Expériences de la vieillesse en établissement à deux siècles d'intervalle : l'humanisation en question ", Genèses, I, 20I7, p. 30-49.

5I | Pilloud, «La thérapeutique par le travail... », art. cité.

52 Pilloud, Julie Hofmann, op. cit. 
affectant leur raison - puisque l'institution n'admet pas de personnes démentes-, mais à cause du désordre social qu'ils pourraient créer. Il convient de préciser que cet asile cantonal, contrairement à quelques maisons de retraite huppées pour pensionnaires fortunés ${ }^{53}$, reçoit avant tout des individus issus de milieux ruraux défavorisés sur le plan économique et socioculturel. Les questions du droit à l'autodétermination ou du besoin d'autonomie sont encore loin des préoccupations institutionnelles, surtout en ce qui concerne les hommes célibataires ou veufs livrés à eux-mêmes, dont les directions institutionnelles redoutent le penchant pour la boisson.

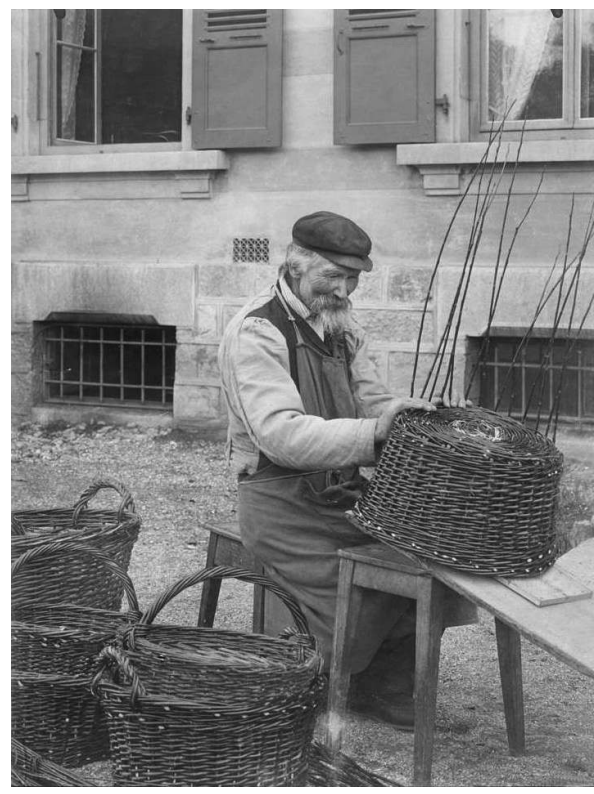

Homme chargé d'activités de vannerie Archives de La Rozavère (photo : de Jongh)

Comment les entraves à la liberté liées au mode de vie institutionnel sont-elles perçues par les vieillards? Les rares occurrences qui donnent à lire le point de vue des personnes âgées, notamment des doléances, ne sont pas relayées dans les rapports annuels de l'institution mais peuvent être documentées dans les procès-verbaux des séances du comité de fondation ou dans des pièces de correspondance entre les familles et la direction. Plusieurs pensionnaires ou certains de leurs proches se plaignent de la sévérité excessive de la directrice, dont le comportement renvoie à la nature des rapports sociaux entre riches et pauvres; une telle attitude se trouve d'ailleurs accrue dans la bienfaisance telle que les milieux philanthropiques la concevaient à l'époque ${ }^{54}$, à savoir basée sur une action de soutien et d'éducation relativement dirigiste. Les membres du conseil institutionnel estiment à l'inverse que la « sévérité [...] est nécessaire.

53 | Nicolas Nussbaum, "L'asile distingué et l'asile des miséreux à Genève au XIxe siècle ", dans Heller, Le poids des ans, op. cit., p. 95-III.

54 | Thierry Christ, «De la Bienfaisance privée à l'Etat social ? Mise en place, financement et contrôle du réseau hospitalier et institutionnel (orphelinats, hospices) à Neuchâtel (I8I5-1914) ", Revue historique neuchâteloise, I, I997, p. 23-5I ; Dumons, "Vieillesse et "État providence" en Suisse romande... ", art. cité ; id., "Genèse d'une politique de vieillesse en Suisse (Fin XIX'-1947) ", Cahiers de l'IDHEAP, 97, 1993; id., "Vieillesse et assistance à Genève entre les deux guerres : les budgets du Bureau central de bienfaisance et du comité cantonal de Pro Senectute ", Revue du Vieux Genève, 1998, p. I2I-I25 ; Kurt Seifert, Chronique Pro Senectute. De l'aide aux indigents au soutien à toutes les personnes âgées, Zürich, Pro Senectute, 2007. 
N'oublions pas qu'elle [la directrice] a affaire à des hommes et des femmes dont le passé n'a pas été une préparation à une vie réglée, ordonnée ${ }^{55}$ ".

Face à des vieillards qui réclament davantage de liberté de mouvement et une possibilité de sortir de l'asile, l'équipe de direction acte du fait que les autorisations ne peuvent être délivrées que si la famille invite un pensionnaire à la maison, les absences ne devant pas excéder une journée par semaine. Un point qui est d'ailleurs spécifié dans le règlement intérieur de l'asile, lequel prévoit les heures des repas et de repos de façon stricte et démontre une forte régulation du quotidien institutionnel. À nouveau, comme dans les modèles institutionnels de l'époque - asile d'aliénés, maison de redressement, ou encore home pour handicapés -, ce sont davantage les besoins de l'institution qui priment, dès lors que celle-ci fonctionne avec un personnel très limité par rapport au nombre de pensionnaires ${ }^{56}$. Toute modification des rituels journaliers impliquerait un surcroît de travail et de coûts.

De manière générale, l'asile de vieillards ne parvient pas à répondre à toutes les demandes d'admission qui lui sont présentées, malgré la création progressive d'autres hospices similaires sur le territoire vaudois à partir de la fin des années $1890^{57}$. L'opportunité d'un agrandissement commence à se faire sentir peu de temps après l'ouverture de l'établissement. Régulièrement, devant l'afflux des requêtes d'entrée et l'âge grandissant des vieillards accueillis, les institutions s'efforceront d'adapter les mesures qu'elles entendent fournir en induisant des transformations. Lorsqu'un déménagement s'avérera nécessaire, l'asile de La Rozavère optera pour des locaux non seulement plus nombreux mais aussi plus grands, ce qui lui permettra d'être plus en conformité avec l'évolution des seuils de sensibilité face à la notion d'intimité et de promiscuité, en mettant fin $\mathrm{au}$ « quasi-entassement dans lequel avaient vécu les vieillards » jusqu'ici ${ }^{58}$.

\section{Les mutations sociales et institutionnelles de la première moitié $\mathrm{du} \mathrm{xx}^{\mathrm{e}}$ siècle}

Le début $\mathrm{du} \mathrm{xx}^{\mathrm{e}}$ siècle augure une série de modifications qui auront des incidences sur la perception des besoins des vieillards et les modalités de leur prise en charge. Peu à peu, les personnes âgées sortent du système d'aide charitable privée pour accéder à des prestations d'assistance légale ${ }^{59}$. En France,

55 | Archives de La Rozavère, extrait du PV de la séance de direction du I $^{\text {er }}$ juillet I893.

56 | Pilloud, Julie Hofmann, op. cit. ; Feller, Histoire de la vieillesse en France..., op. cit.

57 | Heller, Le poids des ans, op. cit.

58 | Archives de La Rozavère, Rapport annuel 1900.

59 | Tabin, Frauenfelder, Togni et Keller, Temps d'assistance, op. cit. 
dès I905, une loi rend obligatoire l'assistance aux vieillards indigents ${ }^{60}$. Cette disposition législative aura comme effet d'englober non pas principalement les " cas sociaux » en marge de la société, mais tout citoyen âgé vis-à-vis duquel la société se met à penser qu'elle a une dette sociale. L'État allemand a été précurseur sur ce point puisqu'une assurance invalidité et vieillesse a été mise en place par le chancelier Bismarck depuis $1889^{61}$. Désormais, les personnes âgées se voient attribuer des droits à des prestations d'aide, sans être tributaires de secours conditionnés à un certain mérite. Ces changements sociaux auront un fort impact sur l'image sociale des seniors, rendant ceux-ci progressivement moins redevables mais davantage en capacité de revendiquer ${ }^{62}$. En Suisse, les dispositions législatives se résument à l'entrée en vigueur, en I9I4, de l'assurance maladie obligatoire ${ }^{63}$. Une personne, quels que soient son âge et son revenu, a désormais accès à des soins ; en tant qu'assurée, elle a le droit d'être malade et dans l'incapacité de travailler sans que sa situation financière en soit péjorée. Or, avec l'allongement de l'espérance de vie, de plus en plus de vieillards présentent des affections liées au grand âge, une évolution à la fois démographique et épidémiologique qui jouera un rôle considérable sur le devenir des asiles de vieillards ${ }^{64}$. Ainsi, dès 1903, la direction de La Rozavère commence-t-elle à envisager un projet de construction d'une annexe spécifiquement destinée aux individus devenus invalides ou atteints de maladie aiguë. Les responsables de l'institution reconnaissent véritablement le besoin de cette catégorie de personnes âgées de bénéficier de soins davantage médicalisés et appropriés à leur état de santé, du point de vue physique ou psychique. Les pertes cognitives commencent en effet à être perçues comme des situations convoquant des mesures particulières. Une prise de conscience qui aura plus tard de considérables répercussions sur la formation du personnel affecté à la prise en charge sanitaire : « La division B devenant de plus en plus un hôpital d'incurables, il est nécessaire qu'une garde-malade diplômée soit mise à sa tête pour que l'ordre règne autant que possible ${ }^{65}$ ».

60 | Feller, Histoire de la vieillesse en France..., op. cit.

6I Hans-Joachim Von Kondratowitz, "The Medicalization of Old Age: Continuity and Change in Germany from the late Eighteenth to the early Twentieth Century ", dans Life, Death, and the Elderly..., op. cit.

62 Geneviève Arfeux-Vaucher, "Vieillesse et représentations sociales de la fin du xix siècle à nos jours ", Gérontologie et société, 64, 1993, p. 6I-75; Anne-Marie Guillemard, " Jalons pour une sociologie des politiques sociales : le cas de la politique française de la vieillesse ", Sociologie et sociétés, I6/2, 1984, p. II9-I28; Geneviève Heller, « Société et vieillissement : l'histoire des changements dans notre siècle », dans Vieillir au XXI siècle : continuités et mutations, Lucerne, Société suisse de gérontologie, 2000, p. 16-28.

63 Matthieu Leimgruber, « De la vieillesse-dénuement à la vieillesse assurée : l'invention de la retraite », dans $A u$ fil du temps, op. cit., p. 44-47.

64 | Santos-Eggimann, «Vieillissement et fragilité - comprendre pour prévenir », art. cité.

65 | Archives de La Rozavère, Rapport annuel, 1928. 
Pour l'heure, c'est toutefois le besoin de séparer les groupes de pensionnaires en fonction de leur condition de santé qui prime, afin de fournir des soins et un environnement mieux différenciés :

La plupart de nos vieux ont besoin de tranquillité ; il faut leur éviter toute émotion tant la raison de quelques-uns est chancelante et il suffit de la présence au milieu d'eux d'une ou d'un camarade agité pour les déranger complètement et mettre leur cervelle à l'envers ${ }^{66}$.

La vieillesse indigente et isolée est désormais clairement distinguée de la vieillesse souffrante. $\mathrm{Si}$, pour la seconde, la priorité est plus nettement axée sur les « soins dévoués ${ }^{67}$ » que réclame leur santé, les individus accueillis au motif de leur isolement familial renvoient à la fonction sociale de l'asile. Celui-ci a vocation à « les entourer d'un bras maternel pour calmer leurs angoisses et leurs souffrances $^{68}$ ». Les discours n'hésitent pas à utiliser un champ lexical affectif, qui serait sans doute vu comme excessivement paternaliste et insuffisamment distancé de nos jours. Le besoin de réconfort est évoqué en mettant en avant le rôle central tenu par une aide spirituelle ou religieuse, rendue d'autant plus profitable à l'aube de la mort : les employés de l'asile doivent « apaiser [les vieillards], les encourager, [...] les engager à porter leurs regards sur les sommets d'où viendra le secours ${ }^{69}$ ». La fin de vie est une thématique mentionnée de manière récurrente, reflétant à la fois une vision laudative et transcendante de la mort, tout autant que l'état de la science médicale de l'époque face à une réalité épidémiologique que les professions de santé n'ont pas encore réussi à investir avec un projet curatif ou réadaptatif.

\section{Évolution des représentations et pratiques de prise en charge au milieu $\mathrm{du} \mathrm{Xx}^{\mathrm{e}}$ siècle}

Le tournant décisif dans l'évolution des politiques sociales et sanitaires en faveur de la vieillesse s'amorce avec l'entrée en vigueur des assurances vieillesse, vers le milieu du Xx $x^{\mathrm{e}}$ siècle $^{70}$. En France, le droit à la retraite, prévu depuis I9Io pour les paysans et les ouvriers, sera élargi à l'ensemble des travailleurs dès $1945^{71}$. Deux ans plus tard, la confédération suisse entérine à son tour, après de

66 | Archives de La Rozavère, Rapport annuel, 1920.

67 | Archives de La Rozavère, Rapport annuel, 1913.

68 | Archives de La Rozavère, Rapport annuel, 1932.

69 | Archives de La Rozavère, Rapport annuel, I913.

70 | Pierre Laroque, "Histoire de la politique de la vieillesse en France ", Gérontologie, I3I, 2004, p. 5-I3 ; Henri Péquignot, «La protection sociale des personnes âgées jusqu’à la deuxième guerre mondiale ", Gérontologie, I30, 2003, p. 38-4I.

7I | Feller, Histoire de la vieillesse en France..., op. cit. 
nombreux débats, l'assurance vieillesse et survivants (AVS) ${ }^{72}$. Cette assurance, réclamée depuis $\mathrm{I} 889$, a été repoussée à plusieurs reprises, en raison de facteurs contextuels et de priorités décrétées plus urgentes ${ }^{73}$. Les deux guerres mondiales ont notamment induit des difficultés sociales et économiques dictant d'autres mesures de la part des autorités publiques. Au sortir du second conflit mondial, la conjoncture est plus propice ; lors des Trente Glorieuses qui suivront, des ressources complémentaires seront allouées aux aîné·e·s ${ }^{74}$. En Suisse, la généralisation du système des caisses de retraite et du deuxième pilier à partir des années 1960 représentera une mesure de prévoyance significative pour les salariés, en particulier pour les hommes, puisque les femmes ont encore relativement peu accès au travail à cette époque ${ }^{75}$. Parallèlement, toutes les personnes âgées disposeront de rentes augmentées grâce aux prestations complémentaires ; l'assurance invalidité, entrée en vigueur en I96I, soutiendra quant à elle les individus se trouvant en situation de handicap avant la sortie de la vie active ${ }^{76}$.

Globalement, la période de prospérité qui suit 1945 et l'entrée en vigueur des assurances sociales induisent une modification de la perception du seuil de confort, tant au sein de la population que dans les institutions ${ }^{77}$. Dans la "Maison des vieillards » de La Rozavère (l'institution change de nom et de raison sociale en I954), un effort est fait en particulier sur la réfection des bâtiments, l'accessibilité des commodités ou encore sur la diversification de l'alimentation. La direction relève à ce titre que « la revalorisation des rentes AVS et AI sera en fait tout bénéfique pour les intéressés puisqu'elle permettra d'accorder aux pensionnaires un peu plus de bien-être en raison de l'augmentation du prix de pension $^{78}$ ".

Cette élévation du niveau de vie en général se traduit également dans la modification du tissu économique, avec en particulier l'expansion du secteur tertiaire et le développement du travail des femmes. Autant de changements qui induisent à leur tour des transformations au niveau des structures familiales : ainsi, dans le canton de Vaud, la famille passe progressivement du modèle étendu en milieu agraire à une cellule nucléaire urbaine et salariée ${ }^{79}$. Ces mutations historiques des sociétés modernes impacteront inévitablement les représentations et attitudes face à la vieillesse dépendante, qui plus est à

72 Heller, Le poids des ans, op. cit.

73 | Leimgruber, « De la vieillesse-dénuement à la vieillesse assurée... », art. cité.

74 | Jean-Claude Henrard et Joël Ankri, Vieillissement, grand âge et santé publique, Rennes, École nationale de la santé publique, 2003.

75 | Leimgruber, « De la vieillesse-dénuement à la vieillesse assurée... », art. cité.

76 | Tabin, Frauenfelder, Togni et Keller, Temps d'assistance, op. cit.

77 | Heller, Le poids des ans, op. cit.

78 | Archives de La Rozavère, Rapport annuel, 196I.

79 | Heller, Historique des maisons de retraite..., op. cit. 
l'égard des individus fragilisés psychiquement ${ }^{80}$. Comme le souligne un rapport annuel de La Rozavère en 1956 déjà, « les jeunes ménages ont souvent de la peine à trouver des logements adaptés à leurs moyens ; ils ont assez de peine à loger leurs enfants, les appartements étant de plus en plus petits, pour qu'ils puissent assumer la charge dans son aspect pratique, matériel, moral d'un vieillard sénile ou malade ${ }^{81}$ ». Dès lors, les institutions seront amenées à poursuivre leur processus de médicalisation pour répondre aux besoins sanitaires de pensionnaires toujours plus âgés et atteints dans leur autonomie ${ }^{82}$. Parallèlement, la discipline gériatrique s'orientera vers la recherche de traitements spécifiques aux affections du grand âge, tandis que la gérontologie favorisera une modification du regard porté sur les personnes âgées, en mettant l'accent sur les moyens propres à promouvoir un vieillissement plus autonome ${ }^{83}$.

À partir des années I960, les institutions cherchent à fournir des « loisirs actifs » capables de stimuler les ressources des pensionnaires tout en agrémentant leur quotidien ${ }^{84}$. Peu à peu, d'autres professionnels viendront collaborer avec les soignants de La Rozavère afin de mettre sur pied des animations ; en I965, on fera venir une " assistance sociale pour distraire [les personnes âgées] et les entendre raconter leurs petites histoires ${ }^{85}$ ». En I97I, c'est une ergothérapeute qui fait son entrée dans l'institution, et trois ans plus tard, on parle spécifiquement du secteur de l'animation. Les acteurs sociaux impliqués dans la prise en charge de la vieillesse se diversifient, à la mesure des nouveaux enjeux sociaux et sanitaires reconnus par la société à l'égard de ses vieux ${ }^{86}$. Les conditions d'hébergement sont régulièrement revues à la hausse en fonction de l'évolution des perceptions quant aux droits des personnes âgées à bénéficier d'un espace privé, d'une certaine intimité et d'une « qualité de vie » en institution, ce terme apparaissant pour la première fois dans les années $198 \mathrm{O}^{87}$.

80 | Thierry Samain, Les représentations sociales et culturelles des aînés, Bruxelles, Kluwer, 2002.

8I | Archives de La Rozavère, Rapport annuel, 1956.

82 | Heller, Historique des maisons de retraite..., op. cit.

$83 \mid$ Graham Mulley, «A History of Geriatrics and Gerontology », European Geriatric Medicine, 3/4, 20I2, p. 225-227.

84 | Giovanna Bernard, "D'hier à aujourd'hui, des pratiques d'animation en pleine évolution ", Gérontologie et société, I, 200I, p. 3I-4I ; Pilloud, « La thérapeutique par le travail... », art. cité.

$85 \mid$ Archives de La Rozavère, Rapport annuel, 1965.

86 | Heller, Historique des maisons de retraite..., op. cit. ; ead., "Les lieux de la maladie : quelques repères historiques ", dans Hermann-Michel Hagmann et Jean-Pierre Fragnière (éd.), Maintien à domicile. Le temps de l'affirmation, Lausanne, Réalités sociales, 1997, p. 15-25; Cornelia Hummel, « La construction sociale de la vieillesse : les XVII ${ }^{\mathrm{e}}, \mathrm{XIX}^{\mathrm{e}}$ et $\mathrm{xx}^{\mathrm{e}}$ siècles ", dans Jean-Pierre Fragnière, François Höpflinger et Valérie Hugentobler (éd.), La question des générations. Dimensions, enjeux et débats, Sion, INAG, 2002.

87 | Gabriele Doblhammer et Rembrandt Scholz (éd.), Ageing, Care Need and Quality of Life: The Perspective of Care Givers and People in Need of Care, Wiesbaden, VS Verlag für Sozialwissenschaften, 2010. 
En résumé, on relève, dans l'analyse thématique des rapports annuels sur la longue durée, une véritable inflexion du discours entre les dernières décennies $\mathrm{du} \mathrm{XIX}^{\mathrm{e}}$ siècle et la fin du $\mathrm{Xx}^{\mathrm{e}}$ siècle. Dans les déclarations d'intention - qui ne reflètent certes pas nécessairement la réalité institutionnelle - il y a clairement un changement de perspective entre les besoins de l'établissement et ceux des résident·e·s : si les premiers sont considérés comme primordiaux à une époque où les rares asiles existants doivent fonctionner avec un personnel peu nombreux et pour ainsi dire sans formation, c'est à l'inverse la structure d'accueil qui devrait « s'adapter aux besoins et désirs des pensionnaires ${ }^{88}$ ", si l'on en croit les écrits produits à partir des années 1980 . On prône une « qualité de vie et un cadre de vie dignes d'eux et qui correspondent à leurs attentes », ainsi que du « temps pour l'écoute et le dialogue ${ }^{89}$.

La lecture de telles lignes laisse imaginer un progrès linéaire et continu, qui va nécessairement vers une prise en compte toujours plus pointue et individualisée des besoins des personnes âgées en institution. La situation est toutefois beaucoup plus complexe. Comme a cherché à le démontrer cette étude historique, les représentations et pratiques à l'égard du grand âge varient en fonction de déterminants socioculturels et économiques. Plus précisément, le secteur sociosanitaire est régulièrement lésé lorsque les finances publiques sont moins favorables, les acquis étant susceptibles d'être remis en cause. La crise financière des années I980 conduira nombre de pays, à l'instar de la Suisse, à afficher une inquiétude marquée devant l'élévation constante des coûts de la santé ${ }^{90}$. S'ensuivra une longue période d'austérité budgétaire induisant une rationalisation des prestations et des établissements de soins, ainsi qu'un démantèlement d'une partie du paysage hospitalier. Dans le canton de Vaud, la politique affichée visera clairement à freiner l'augmentation des lits pour personnes dépendantes en promouvant le maintien à domicile ${ }^{91}$. L'intérêt des études sociohistoriques réside précisément dans la mise en lumière de continuités ou au contraire de ruptures en ce qui concerne les modalités de prise en charge des vieux dans la société. Or, un des leitmotivs qui se dégage des recherches diachroniques réside dans cette oscillation entre un processus d'institutionnalisation présenté comme favorable, généralement quand la conjoncture le parait aussi, et une volonté de maintenir ou de ramener au plus vite les individus institutionnalisés dans le milieu communautaire, avec un discours privilégiant la préservation de

88 | Archives de La Rozavère, Rapport annuel, I982.

89 | Ibid.

90 | Gabriella Pedroni et Peter Zweifel, Vieillissement, santé, coûts de santé, Bâle, Pharma Information (Économie et santé), I989.

9I | Hagmann et Fragnière, Maintien à domicile..., op. cit. 
l'autonomie et l'aide informelle ou extra-hospitalière. À l'aube du xxI ${ }^{\mathrm{e}}$ siècle, cet effort de compression des frais de santé se traduira jusque dans la gestion des $\mathrm{EMS}^{92}$, désormais largement subventionnés par les finances cantonales et les prestations des assurances maladie. Ceux-ci sont aujourd'hui sommés de fonctionner de manière rentable, quitte à ce que l'engagement de professionnels formés soit ramené au minimum nécessaire pour permettre aux institutions de répondre à leurs missions médico-sociales. On le voit, les besoins des aînés sont toujours appréhendés dans un horizon mouvant de possibilités, au sein duquel les besoins de la société et les besoins des institutions sociosanitaires doivent également être pris en·considération.

\section{Conclusion}

Les enjeux actuels liés au vieillissement démographique méritent d'être interrogés à la lumière des siècles passés ${ }^{93}$. L'histoire met en effet en évidence les facteurs pesant de tout leur poids sur les visions relatives aux personnes âgées et à leurs besoins, des perceptions qui sont clairement le produit de constructions sociohistoriques en constante mutation ${ }^{94}$. Elle montre à quel point les discours sur les populations vieillissantes sont inscrits dans un contexte où le type d'organisation sociale, politique et économique, ainsi que les références culturelles ne sauraient être ignorés. Une étude sur la longue durée exhorte donc à prendre la mesure des transformations qui se dessinent sur ces divers niveaux afin de répondre aux défis à venir. La perspective diachronique favorise une réflexion critique et nous invite à complexifier et à historiciser les questions relatives aux besoins des seniors. Il serait par ailleurs du plus grand intérêt de favoriser des approches analytiques et des corpus archivistiques qui donnent à lire et à comprendre le point de vue des personnes âgées

92 Laroque, « Histoire de la politique de la vieillesse en France », art. cité ; Péquignot, « La protection sociale des personnes âgées... ", art. cité; Alan Walker, The Politics of Old Age in Europe, Buckingham, Open University Press, 1999.

93 | Hermann-Michel Hagmann, «Vieillesses d'hier et d'aujourd'hui. Émergence d'une problématique socio-démographique ", dans Michel Loriaux, Dominique Remy et Eric Vilquin (éd.), Populations âgées et révolution grise. Les hommes et les sociétés face à leurs vieillissements, Louvainla-Neuve, Ciaco, 1990, p. 575-592.

94 | Pat Thane, The Long Story of Old Age, Londres, Thames \& Hudson, 2005 ; Séverine Pilloud, « Les soins aux personnes âgées : du passé au présent... quel futur ? ", Journal La Source, 125/I, 2015, p. 4-5. 
HMS 12 - 2017 | Médicalisation de la sexualité

elles-mêmes sur leurs propres besoins, des voix qui restent encore trop souvent silencieuses dans les sources historiques ${ }^{95}$.

95 Une recherche, financée par le RECSS et menée par la professeure Dr. Séverine Pilloud et Dr. Delphine Roulet-Schwab en co-requérante, s'est déroulée de novembre 2013 à fin 2014 et s'intitulait : Histoire des discours et pratiques relatifs aux droits des patient-e.s et résident.e.s âgé.e.s (1970-20I4). Regards croisés de différents acteurs sociaux et institutionnels sur l'évolution des droits et leurs enjeux éthiques (étude exploratoire centrée sur un EMS du canton de Vaud). Elle avait comme principal objectif d'examiner les points de convergence ou de divergence entre les regards des différents protagonistes sur les droits des personnes âgées en EMS. Une autre recherche, exploratoire, a été financée par la fondation Leenaards (2016). Elle a pour titre: Dimension relationnelle de l'accompagnement soignant et incidence sur la qualité de vie perçue par les personnes âgées : perspectives sociohistoriques et psychosociologiques. Elle est également menée par la professeure Dr. Séverine Pilloud et Dr. Delphine Roulet-Schwab est la co-requérante. 